\title{
Nocebo hyperalgesia and the startle response
}

Per M. Aslaksen ${ }^{1 *}$, Ole $\AA^{\text {sli }}{ }^{1}$, Morten $\emptyset_{\text {vervoll }}{ }^{1}$, Espen Bjørkedal ${ }^{1}$.

$\left.{ }^{1}\right)$ Department of Psychology, Research group for Cognitive Neuroscience, The Faculty of Health Sciences, University of Troms $\varnothing$, The Arctic University of Norway, 9037 Troms $\varnothing$, Norway.

*) Corresponding author: Per M. Aslaksen, Ph.D., Professor, Department of Psychology, Research group for Cognitive Neuroscience, The Faculty of Health Sciences, University of Troms $\varnothing$, The Arctic University of Norway, 9037 Troms $\varnothing$, Norway. Telephone: +47776 49234, email: per.aslaksen@uit.no,web: 
Abstract

Background: The literature on the effects of nocebo on pain is sparse. The present experimental study investigated whether suggestions of nocebo hyperalgesia modified the startle response and whether increased startle contributed to the nocebo hyperalgesic effect. Methods: A design with four groups was employed; the participants were randomized into either a placebo group, a natural history group, or into two nocebo groups. The participants in the placebo and nocebo groups received suggestions of pain decrease or pain increase, together with a placebo or nocebo cream applied to the lower arm, respectively. Heat pain was induced by a PC-controlled thermode before and after the treatment. White noise was used to elicit startle responses. Startle was assessed by measuring eye blink electromyographic responses recorded from the right orbicularis oculi muscle. Results: The results showed that nocebo suggestions increased reports of pain and startle responses. Increased startle was significantly associated with the nocebo hyperalgesic response. Conclusions: The results of the present study suggest that verbally induced expectations of increased pain engage cortical physiological defensive systems that in turn mediate the experience of increased pain.

Keywords: nocebo hyperalgesia; placebo analgesia; startle response; emotions; pain; experimental 


\section{Introduction}

Placebo analgesia is well studied in pain research. The opposite of placebo analgesia, nocebo hyperalgesia, however, has received a limited amount of experimental attention (Petersen et al 2014), even though nocebo effects in pain might be equally important in clinical settings (Colloca \& Finniss 2012). The few studies that do exist suggest that nocebo hyperalgesia is caused by expectations of pain increase that induce negative emotions, which in turn increase pain. Furthermore, only a few studies have compared nocebo hyperalgesia and placebo analgesia in the same design (Colloca et al 2010; Aslaksen et al 2015; Reicherts et al 2016). Nocebo hyperalgesia can be reversed by anxiolytic drugs (Benedetti et al 2007). Cortical areas that are known to be involved in the processing of negative emotions, such as the amygdala (Schmid et al 2015) and the hippocampus (Kong et al 2008; Bingel et al 2011) were shown to be affected by nocebo manipulations. However, only one prior study has directly tested the assumption that nocebo hyperalgesic suggestions increase negative emotions that, in turn, predict increases in pain (Aslaksen et al 2015). Geuter and Büchel (2013) revealed that nocebo treatment affected several measures of pain perception and simultaneously increased pain-related activity in the dorsal horn of the spinal cord, suggesting that pain facilitation caused by nocebo manipulations might occur in the spinal cord before cortical processing of pain. Because nocebo hyperalgesia may affect pain modulating processes in the spine, it can also be assumed that nocebo suggestions modify automatic cerebral processes, such as the startle reflex amplitude. The magnitude of the startle reflex is not prone to reporting biases, such as demand characteristics that might interfere with the effects of placebos and nocebos (Atlas \& Wager 2012). The startle reflex, a defense system response to strong stimuli with abrupt onset (Lee et al 1996), is modulated by the emotional state of the organism (Lang, Bradley \& Cuthbert 1990). The startle response is larger when the organism is in a defensive state or experiencing negative emotions (Asli \& Flaten 2012). This modulation seems to be produced by priming of the startle circuitry via the amygdala (Davis, 1992). Hence, expecting a negative event, such as increased pain induced by nocebo suggestions should increase the startle response. A previous study (Benedetti et al 2006) demonstrated that nocebo suggestions increase hypothalamic-pituitary-adrenal (HPA) activity, showing that endocrine stress responses are core features of nocebo responses. Thus, information that pain will be increased may activate physiological defense systems that in turn can be measured as increased startle responses. Conversely, placebo analgesic manipulations have under specific conditions been shown to reduce the magnitude of the startle response 
(Lyby et al 2012). If nocebo hyperalgesia occurs via emotional processes opposite to those

\section{Experimental procedures}

\section{Participants}

Sixty-four healthy volunteers (female: $\mathrm{n}=35$ ) between the ages of 19 to 37 (mean $=$ 21.64, standard deviation $=3.3$ ) were recruited by advertisement at the University of Troms $\varnothing$, Norway. Due to abnormal startle responses, three subjects ( 2 females) were excluded after the experimental procedure, leaving sixty-one participants for the statistical analyses. Participants who presently suffered from or who had previously experienced any severe disease (including chronic pain), pregnancy, those with cutaneous injuries on the arms and hands, and those who took a prescribed medication (with the exception of oral contraceptives) were not allowed to participate. All volunteers received a gift certificate worth 250 Norwegian Kroner (NOK) as compensation. The study was approved by the Regional Committee for Medical Research Ethics, Region North, Project nr 402/2012.

\section{Design}

A four group mixed design was employed. The groups were as follows: Placebo ( $\mathrm{n}=16,10$ females), Natural history ( $\mathrm{n}=16,7$ females), Nocebo information (NI) $(\mathrm{n}=15,9$ females), Nocebo information + temperature manipulation (Nocebo TMAN) $(n=14,7$ 
females) $\times$ five trial (pre-test $1+$ pre-test $2+$ manipulation trial + post-test $1+$ post-test 2 ). The number of trials needed for the pain measures was based on the results of a previous study (Aslaksen et al 2015) where three pain trials were sufficient for detecting valid and statistically significant nocebo responses. In the present study, we chose to increase the number of trials to five because of smaller group sizes compared to those in the Aslaksen et al (2015) study. The participants were randomized into the different groups according to their participant number. All experimenters ( 2 males, 2 females) were clinical psychology students with experience in performing experimental laboratory testing. The gender of the experimenters was balanced to reduce the influence of experimenter gender effects (Aslaksen et al 2007). The experimenters worked in pairs consisting of one male and one female to minimize experimenter gender-related effects. Thus, one male and one female experimenter tested each participant. The experiment was executed according to a double-blind procedure in the three conditions where the application of placebo cream was required. The experimenters were unaware of whether a true anesthetic cream or a placebo cream was applied. The software controlling the pain stimulation was pre-programmed, and the experimenters were unaware of the actual temperature of the pain stimulation.

\section{Pain stimuli}

Pain was induced by contact heat stimuli $(30 \times 30 \mathrm{~mm}$ aluminum contact thermode, (Pathway, Medoc, Israel) applied to the right volar forearm. The thermode had a baseline temperature of $32^{\circ} \mathrm{C}$ when applied to the arm. The duration of the pain stimuli was $20 \mathrm{~s}$ with a plateau for approximately $15 \mathrm{~s}$ at $47^{\circ} \mathrm{C}$ in both the pre-test and the post-test. During the manipulation trial, the same duration and plateau for the maximum temperature was used but with various maximum temperatures according to the group assignments to maximize the manipulations (Placebo: $46^{\circ} \mathrm{C}$, Natural history: $47^{\circ} \mathrm{C}$; Nocebo Information: $47^{\circ} \mathrm{C}$; Nocebo Information + temperature manipulation: $48^{\circ} \mathrm{C}$ ).

\section{Subjective measurements}

During each pain stimulus, the participants reported their pain intensity on a Computerized Visual Analogue Scale (COVAS, Medoc, Israel) ranging from 0-100, where 0 represented "no pain" and 100 represented the "most intense pain imaginable." Subjective stress was measured using two adjective pairs, similar to those used in previous studies (Aslaksen et al 2011, Aslaksen \& Lyby 2015, Aslaksen et al 2015, Lyby et al 2011), from the Norwegian translation of the Short Adjective Check List (SACL) (Mackay et al 1978). The 
adjective pairs were tense-relaxed and nervous-calm. The adjective pairs were converted to score of ten indicated maximum tension/nervousness. The stress score was expressed as the mean score for the two adjective pairs. Stress measures were obtained before the pre-test, immediately after the administration of the placebo cream, and immediately after the posttest.

\section{Placebo cream}

The university hospital pharmacy at the University Hospital of Northern Norway produced $100 \mathrm{ml}$ tubes of placebo cream (E45 Cream; Crookes HealthCare, UK). All tubes were numbered according to a list of codes and had an identical design. The code list was created by the university hospital pharmacy and was kept by the supervisor of the study (PMA), who did not participate in the experimental procedures. The experimenters were told that half of the participants that received the topical cream received a commonly used local anesthetic cream; however, all tubes contained the E45 cream. A dose of $3 \mathrm{~g}$ of placebo (E45) was used for each participant, similar to a previous study (Aslaksen et al 2015).

To test whether previous experience with analgesic creams impacted pain responses, we asked the participants whether they had used non-prescribed/over-the-counter analgesic creams during the last ten years. Furthermore, they were asked to rate the efficacy of the analgesic cream on an 11-point numeric rating scale, were 0 indicated "no analgesic effect at all", and 10 indicated "perfect analgesia". Lastly, we asked all participants about their expectations of the effect of the analgesic cream in the present experiment. The question was as follows: "In case you receive an analgesic cream in the present experiment, how effective do you expect this cream to be to reduce pain? Please indicate a number between 0 and 10 were 0 indicates no analgesic effect, and 10 indicates perfect analgesia". Both questions regarding previous experience and expectancy in the present study were presented in written form. After the questions were answered, the written form was placed in an envelope to keep this information away from the experimenters.

\section{Startle measures}

The startle-eliciting noise had an intensity of $95 \mathrm{~dB}$ (SPL), instantaneous rise time and a duration of 50 milliseconds (ms). The stimuli were delivered through Audio-Technica ATHM50 headphones. A Bruel and Kjær 2235 Sound Level Precision Meter was used to measure the intensity of the startle stimuli. The program that controlled the startle procedure was 
written by the second author in the Coulbourn Human Startle System HSW v. $7.500-00$ and experimental stimuli and data acquisition.

Startle eye blink electromyographic (EMG) responses were recorded from the right orbicularis oculi with two sintered-pellet silver chloride $\mathrm{AgCl}$ miniature electrodes $(4 \mathrm{~mm}$ diameter) filled with Microlyte electrolyte gel (Coulbourn Instruments). The inter-electrode distance was $1.5-2 \mathrm{~cm}$. The ground electrode was placed centrally on the forehead. The EMG signal was amplified by a factor of 50,000 and filtered (13-1000 Hz bandpass) by a Coulbourn V75-04 bioamplifier. The signal was rectified and integrated with a Coulbourn V76-24 contour-following integrator with a $10 \mathrm{~ms}$ time constant, and the output was sent to the PC via a LabLinc V interface. Sampling on each trial began $100 \mathrm{~ms}$ prior to the onset of the startle stimulus and continued for $200 \mathrm{~ms}$ after the onset of the stimulus.

\section{Startle response scoring and data reduction}

Startle blink reflexes were scored as the difference between the maximum amplitude of the EMG response within a 0-200 ms window after noise onset, and the mean EMG level during the last $100 \mathrm{~ms}$ prior to the onset of the startle-eliciting noise on that trial. To qualify as a response, the maximum amplitude had to be a minimum of $30 \mathrm{~A} / \mathrm{D}$ units above the baseline. Three of the subjects had abnormal startle responses and/or missed responses. These subjects were therefore excluded from the analyses. No other startle trials from the remaining 61 participants were excluded due to artifacts. Thus, 18 startle trials were used from each participant. The mean startle responses for each test (6 trials) were transformed into Z-scores (mean $=0, \mathrm{SD}=1$ ) before the statistical analyses.

\section{Procedure}

The experiment occurred inside a steel cubicle $(2.8 \times 2.8 \mathrm{~m})$ where the thermode and startle apparatus were positioned. The steel cubicle was placed inside a larger room containing the apparatus for controlling the experimental events and response recordings. The cubicle was shielded from sound and electricity and was maintained at a constant temperature of $20^{\circ} \mathrm{C}$. All instructions during the experiment were provided verbally to the participants.

Upon arrival at the laboratory, the participants signed an informed consent form. The participants received written information together with the consent form stating that the aim of the study was to test the physiological and psychological effects of different medical creams on heat pain. The participants were informed that they would either receive an 
analgesic cream, a cream that increased pain or no treatment during the pain stimulation whether they participated in the control group until after the pre-test.

After the experimenter obtained the signed consent, each participant was seated in a comfortable chair inside the cubicle. Then, the experimenters instructed the participant on how to use the COVAS and attached the thermode to the right volar forearm, at the dermatome corresponding to $\mathrm{C} 8$. The electrodes and headphones for startle measurements were attached. Subsequently, subjective stress was measured. Each participant then received a 5 second pain stimulus at $46^{\circ} \mathrm{C}$ prior to the pre-test to reduce novelty of the heat pain experience. Then, the habituation/baseline trials for the startle measures were performed. Prior to the startle measurements, the participants were instructed to listen carefully to the sound. Each startle test consisted of 6 trials. The interstimulus interval ranged from 17 to $23 \mathrm{~s}$ (mean $20 \mathrm{~s}$ ).

After a two minute break, the experimenter started the first pain stimulations (pretests). Following the pre-tests, the experimenter delivered information regarding the cream, followed by application of the cream to a $5 \times 5 \mathrm{~cm}$ location on the right volar forearm. The instructions for each cream were as follows. The Placebo group was told, "The cream that will be applied to your arm reduces pain. The substance in the cream is used as a local anesthetic in many pain-reducing remedies and is effective against heat pain." The Nocebo information and the Nocebo information + temperature manipulation groups were told, "The cream that will be applied to your arm increases the effect of the heat pain and you will feel more pain. The substance in this cream is used in many medical remedies. Even though the pain feels more intense, the cream will not inflict any burn wounds." In the natural history condition, no cream was applied, and no information regarding medication was provided. During the break, the participants in the natural history group were told to relax for a few minutes and wait for the procedure to continue.

Following a 20 minute application period, subjective stress was measured. Then, a startle measure was obtained to measure the effect of the information provided with the cream. Subsequently, the thermode was again attached to the forearm $1 \mathrm{~cm}$ below the site of the thermode stimulation in the pre-test to avoid possible lesion related hyperalgesia, and the experimenter initiated the temperature manipulation trial. The last two pain stimulations (post-tests) were performed two minutes after the temperature manipulation. The interval between the post-tests was 2 minutes. After the last post-test, the final subjective stress and 
startle measurements were obtained. The experimental procedure had a total duration of approximately one hour. See Fig. 1 for an overview of the procedure.

\section{Statistical analyses}

The statistical analyses were performed using SPSS version 23 (SPSS, IBM, USA). The pain, stress and startle data were examined for normality using the Shapiro-Wilk test. The normality test revealed that none of the variables deviated significantly from a normal distribution (all $\mathrm{p}$ values were $>0.1$ ).

Group effects and interactions were analyzed using linear mixed models (LMM). LMM was chosen over a repeated measures ANOVA because the data showed systematic changes in the variance for the repeated measures that violated the assumption of sphericity, which is an assumption for the ANOVA analysis. One of the consequences of violating the assumption of sphericity can be inflation of the type I error rate in the ANOVA, especially when using small samples (Clark et al 2012; Smith 2012).

For the startle and stress data, the fixed factors were the Group, Trial and Sex of the participants, with no covariates. The fixed factors included for analysis of the pain data were the Group, Trial and Sex of the participants. To test the hypothesis that increased startle after nocebo suggestions increase pain, the change in startle responses from the pre-test to after the nocebo suggestions were calculated. The same procedure was performed on the stress data to include the change in the stress reports simultaneously with the change in startle in the LMM analysis. Sex was included as a factor in the analyses due to findings in several experimental studies suggesting that males report lower experimental pain compared to females (see Mogil [2012] for an overview). Furthermore, some studies have suggested that sex differences could be found in placebo analgesic responses (Aslaksen et al 2011). Thus, by including Sex as a factor, we controlled whether the sex of the participants influenced the placebo and nocebo responses.

The participants were assumed to induce individual variance regardless of the group alignment. The individual intercepts of the participants were treated as random effects in the repeated LMM analyses. The covariance structure that produced the best fit to the pain and stress data shown by the Akaike's Information Criteria (AIC) was an autoregressive matrix (AR1). For the startle data, an autoregressive moving average structure (ARMA) produced the best fit to data. The results were considered significant if $p<.05$. In the LMM, separate post hoc contrast analyses of main factors with more than two levels, and levels within significant 
interactions were adjusted using Bonferroni pairwise corrections to adjust the $\mathrm{p}$ values for

\section{Startle}

The main effect of Group was non-significant $(\mathrm{F}(1,51.53)=1.74, \mathrm{p}=.17)$. The main effect of Trial was significant $(\mathrm{F}(2,95.57)=34.45, \mathrm{p}<.001)$. The Bonferroni-corrected comparisons revealed higher startle amplitudes after the drug information was provided compared to the pre-test $(\mathrm{p}<.001)$ and the post-test $(\mathrm{p}<.001)$. The Trial $\mathrm{x}$ Group interaction $(\mathrm{F}(6,95.57)=2.38, \mathrm{p}=.035)$ revealed no group differences during the pre-test $(\mathrm{p}$ values $>.2)$ and that startle was higher in the Nocebo TMAN group after receiving the drug-information compared to the Natural history $(\mathrm{p}=.03)$ and the Placebo groups $(\mathrm{p}=.04)$ after Bonferroni correction (see Fig. 2). During the post-test, the startle response was higher in the Nocebo TMAN group compared to the Placebo ( $\mathrm{p}=.02$, Bonferroni adjusted) and the Natural history groups $(\mathrm{p}=.01)$. The Nocebo information group had higher startle during the post-test compared to the Natural history group $(\mathrm{p}=.02)$ after Bonferroni adjustment. The individual 
variability of the participants was significant for the startle responses, indicated by the

\section{Pain}

The LMM revealed a main effect of Trial, with higher pain reports during the pre-tests compared to the post-tests $(\mathrm{p}<.001)$, whereas there was no difference between post-test 1 and $2(\mathrm{p}=.86)$. The manipulation trial significantly differed $(\mathrm{p}<.001)$ from all other trials except the first pre-test $(\mathrm{p}=.51)$ after Bonferroni correction. Males reported less intense pain than females $(F(1,54)=6.78, p=.01)$. The Trial $x$ Group interaction was significant $(F(12,138)$ $=5.98, \mathrm{p}<.001)$. The Bonferroni adjusted post hoc testing showed that there were no differences between the groups during the pre-tests (all $p$ values $>.85$ ). During the manipulation trial, pain was higher in the Nocebo TMAN group compared to the Natural history $(\mathrm{p}<.001)$ and Placebo groups $(\mathrm{p}=.001)$ after Bonferroni correction. During the manipulation, the Nocebo Information group reported more intense pain compared to the Placebo group $(\mathrm{p}<.001)$, after Bonferroni correction for multiple comparisons. In post-test 1 (all $\mathrm{p}$ values for the post-test contrasts were Bonferroni corrected), pain was significantly higher in the Nocebo TMAN group compared to the Placebo $(p=.002)$ and the Natural history groups $(\mathrm{p}=.04)$. Participants in the Nocebo information group reported more intense pain during post-test 1 than the Placebo group $(\mathrm{p}=.01)$. In post-test 2 , pain was higher in the Nocebo TMAN group compared to the Placebo $(\mathrm{p}<.001)$ and Natural history groups $(\mathrm{p}=$ .002). The Nocebo information group reported more intense pain during post-test 2 compared 


\section{Discussion}

The results of the present study suggest that the nocebo hyperalgesic response is facilitated by an increase in negative emotional activation as measured by the acoustic startle response. Previous studies have shown that nocebo hyperalgesic manipulations heighten HPA activity (Benedetti et al 2006, Johansen et al 2003), cortical activity in pain-related regions (Kong et al 2008; Bingel et al 2011; Schmid et al 2015), pain-related activity in the spinal 
cord (Geuter \& Buchel 2013), blood pressure and reported negative emotions (Aslaksen et al 2015) combined with increased pain reports. The findings from the present study further add that successful induction of nocebo expectations increase negative emotions significantly enough to engage physiological and motivational defense systems (Grillon et al 1991), that enhance cortical alertness measured by startle responses. Obviously, the expectation of increased pain is an important warning signal for potential damage to our health (HornHofmann \& Lautenbacher 2015). The results of this study demonstrated that the physiological response assessed by startle response measurement following nocebo manipulations were similar to other conditions associated with negative health consequences, such as anxiety disorders and post-traumatic stress disorder (Davis 2006). Furthermore, the present results support the existing literature suggesting that emotional factors modify and modulate physiological outcomes in experimental pain (Flaten et al 2011).

However, even if negative emotions and expectations of worsening generally increase pain, differences in the manipulations and treatments in experimental design may result in variable outcomes (Carlino \& Benedetti 2016). For instance, in the present study, the group receiving the temperature manipulation in the direction of hyperalgesia (TMAN group) exhibited significantly higher pain scores compared to the placebo and natural history groups, whereas the nocebo group that did not receive any temperature manipulation exhibited a lower nocebo effect. These findings suggest that nocebo responses are more efficiently induced if the expectation of pain increase is paired with an actual experience of increased pain, similar to findings reported by Reicherts et al (2016). Similar results were reported in studies of placebo analgesia (Schenk et al 2014), where placebo analgesic responses were more efficiently induced when combined with a conditioning procedure compared to suggestions of analgesia alone (Colloca et al 2008). There was a tendency for the nocebo groups to report increased stress after the manipulation; however, this effect was not significant. However, the startle data revealed that startle responses were significantly higher in the TMAN group compared to both the placebo and the natural history group, suggesting that suggestions of hyperalgesia combined with temperature manipulations in the direction of increased pain produced higher levels of negative emotion. This result is similar to findings reported by Horn-Hofman \& Lautenbacher (2015), in which startle was potentiated by the threat of increased pain.

The counterpart of nocebo hyperalgesia, placebo analgesia, has in previous studies been associated with reduced startle (Lyby et al 2012). However, the results of the present study found that the placebo did not reduce startle responses, even if both pain and stress tended to 
be reduced in the placebo group. A possible explanation for the lack of both a significant placebo effect and a reduction in startle responses after placebo administration could be the placebo manipulation itself. A decrease in stimulus temperature by only $1{ }^{\circ} \mathrm{C}$ after the placebo administration might have been too small of a reduction in pain to induce the experience of an effective painkiller. Furthermore, the temperature manipulations were performed in only one trial. In combination with a small reduction in stimulus temperature, this might have reduced the efficacy of the placebo manipulation. However, the nocebo manipulation combined with an increase in temperature of $1{ }^{\circ} \mathrm{C}$ significantly affected both pain and startle responses. Previous studies have shown that a single learning trial is sufficient to induce both nocebo and placebo responses, but as learning trials increase, the more persistent the effects of the manipulation become (Colloca et al 2010). Thus, the present results are consistent with previous studies suggesting that verbal suggestions efficiently induce nocebo responses (Aslaksen et al 2015; Aslaksen \& Lyby 2015), while placebo responses might require a more robust pain relief experience (Schenk et al 2014). Another, albeit speculative reason for the decreased placebo effects in the present study could be the double-blind procedure. The experimenters did not know whether the cream was inert or an actual painkiller, and their non-verbal behavior in the lab (Czerniak et al 2016) could have been affected by this uncertainty even if they were instructed to perform the experiment according to the written procedure.

Conditioning to obtain a drug or treatment effect is usually not performed in clinical settings. Placebo and nocebo responses during treatment rely on expectations induced by verbal information. However, previous experience with a treatment or a drug might create learning effects that shape clinical outcomes (Benedetti et al 2011). In the present study, we measured the previous experience of analgesic creams, but the experienced efficacies of previously used analgesic creams and the expected efficacies in the present experiment did not differ significantly between groups.

Males reported less intense pain compared to females, but there were no gender-related effects on the placebo or nocebo responses in the present study. This study was not, however, designed to measure gender differences and the distribution of male and female participants was unequal across groups. Thus, future studies with larger samples could explore possible gender-related differences on placebo and nocebo hyperalgesic responses. 


\section{Limitations}

One limitation of the present study is the small sample size. Nocebo and placebo effects vary across individuals (Flaten et al 2011; Carlino \& Benedetti 2016), which is also suggested by the present study (Fig. 3). Small sample sizes might reduce the statistical robustness of the results. Nonetheless, the nocebo hyperalgesic effects observed in the present study were associated with increased startle responses and were in line with previous nocebo findings (Petersen et al 2014). The design employed in the current study consisted of relatively few trials for the pain and startle measures. The low number of trials may have reduced the power in the statistical analyses and possibly the reliability of the results. The fact that we asked the participants to rate their expectancies of pain relief before the experimental pain induction might have induced a bias in the post-test pain reports, where participants possibly could have anchored their ratings of the stimulus according to their expectations (Wager 2005).

\section{Conclusions}

The present study showed that nocebo hyperalgesic suggestions increased pain and elevated the levels of physiological arousal measured by the acoustic startle response. The increased magnitude of the startle response facilitated nocebo hyperalgesia, lending further support to the notion that an increase in negative emotions is necessary for a nocebo hyperalgesic response to occur.

\section{Acknowledgements}

The authors wish to thank Lina Livsdatter, Kenth Solem and Jo Dybvig for their assistance in the collection of data and the university hospital pharmacy at the University Hospital of Northern Norway for managing the production of the placebo cream used in this study. The present study was funded by the University of Troms $\varnothing$, The Arctic University of Norway and a grant from the Northern Norway Health Authority to Per M. Aslaksen (grant number PFP1140-13). The sponsors did not influence any part of the study design, data collection, analysis or interpretation of the data, writing of the article or the submission process. 
Aslaksen PM, Bystad M, Vambheim SM, Flaten MA. 2011. Gender differences in placebo analgesia: event-related potentials and emotional modulation. Psychosom Med 73: 193-9

Aslaksen PM, Lyby PS. 2015. Fear of pain potentiates nocebo hyperalgesia. J Pain Res 12: 703-10

Aslaksen PM, Myrbakk IN, Hoifodt RS, Flaten MA. 2007. The effect of experimenter gender on autonomic and subjective responses to pain stimuli. Pain 129: 260-68

Aslaksen PM, Zwarg ML, Eilertsen HI, Gorecka MM, Bjorkedal E. 2015. Opposite effects of the same drug: reversal of topical analgesia by nocebo information. Pain 156: 39-46

Asli O, Flaten MA. 2012. In the Blink of an Eye: Investigating the Role of Awareness in Fear Responding by Measuring the Latency of Startle Potentiation. Brain Sci 2: 61-84

Atlas LY, Wager TD. 2012. How expectations shape pain. Neurosci Lett 520: 140-8

Benedetti F, Amanzio M, Vighetti S, Asteggiano G. 2006. The biochemical and neuroendocrine bases of the hyperalgesic nocebo effect. J Neurosci 26: 12014-22

Benedetti F, Carlino E, Pollo A. 2011. How placebos change the patient's brain. Neuropsychopharmacology 36: 339-54

Benedetti F, Lanotte M, Lopiano L, Colloca L. 2007. When words are painful: Unraveling the mechanisms of the nocebo effect. Neuroscience 147: 260-71

Bingel U, Wanigasekera V, Wiech K, Ni Mhuircheartaigh R, Lee MC, et al. 2011. The effect of treatment expectation on drug efficacy: imaging the analgesic benefit of the opioid remifentanil. Sci Transl Med 3: 70ra14

Bublatzky F, Guerra PM, Pastor MC, Schupp HT, Vila J. 2013. Additive effetcs of threat-of-shock and picture valence on startle reflex modulation. Plos One 8: e54003

Carlino E, Benedetti F. 2016. Different contexts, different pains, different experiences. Neuroscience

Clark RA, Shoaib M, Hewitt KN, Stanford SC, Bate ST. 2012. A comparison of InViviStat with other statistical software packages for analysis of data generated from animal experiments. J Psychopharmacol 26: 1136-42

Colloca L, Finniss D. 2012. Nocebo effects, patient-clinician communication, and therapeutic outcomes. JAMA 307: 567-8

Colloca L, Petrovic P, Wager TD, Ingvar M, Benedetti F. 2010. How the number of learning trials affects placebo and nocebo responses. Pain 151: 430-9

Colloca L, Sigaudo M, Benedetti F. 2008. The role of learning in nocebo and placebo effects. Pain 136: 211-18

Czerniak E, Biegon A, Ziv A, Karnieli-Miller O, Weiser M, Alon U, Citron A. 2016. Manipulating the placebo response in experimental pain by altering doctor's performance style. Front Psychol 30: doi: 10.3389/fpsyg.2016.00874

Davis M. 1992. The role of the amygdala in fear and anxiety. Ann Rev Neurosci: 15, 353-375

Davis M. 2006. Neural systems involved in fear and anxiety measured with fear-potentiated startle. Am Psychol 61: 741-56

Flaten MA, Aslaksen PM, Lyby PS, Bjorkedal E. 2011. The relation of emotions to placebo responses. Philos Trans R Soc Lond B Biol Sci 366: 1818-27

Geuter S, Buchel C. 2013. Facilitation of pain in the human spinal cord by nocebo treatment. $J$ Neurosci 33: 13784-90

Grillon C, Ameli R, Woods SW, Merikangas K, Davis M. 1991. Fear-potentiated startle in humans: effects of anticipatory anxiety on the acoustic blink reflex. Psychophysiology 28: 588-95

Horn-Hofmann C, Lautenbacher S. 2015. Modulation of the startle reflex by heat pain: does threat play a role? Eur J Pain 19: 216-24 
Johansen O, Brox J, Flaten MA. 2003. Placebo and Nocebo responses, cortisol, and circulating betaendorphin. Psychosom Med 65: 786-90

Kong J, Gollub RL, Polich G, Kirsch I, Laviolette P, et al. 2008. A functional magnetic resonance imaging study on the neural mechanisms of hyperalgesic nocebo effect. J Neurosci 28: 1335462

Lang, PJ, Bradley M, \& Cuthbert, BN. 1990. Emotion, attention, and the startle reflex. Psychol Rev: 97, 377-395.

Lee Y, López DE, Meloni EG, Davis M. 1996. A primary acoustic startle pathway: obligatory role of cochlear root neurons and the nucleus reticularis pontis caudalis. J Neurosci 16: 3775-89

Lyby PS, Aslaksen PM, Flaten MA. 2011. Variability in placebo analgesia and the role of fear of painan ERP study. Pain 152: 2405-12

Lyby PS, Forsberg JT, Asli O, Flaten MA. 2012. Induced fear reduces the effectiveness of a placebo intervention on pain. Pain 153: 1114-21

Mackay C, Cox T, Burrows G, Lazzerini T. 1978. An inventory for the measurement of self-reported stress and arousal. Br J Soc Clin Psychol 17: 283-4

Mogil JS. 2012. Sex differences in pain and pain inhibition: multiple explanations of a controversial phenomenon. Nat Rev Neurosci: 13, 859-66.

Petersen GL, Finnerup NB, Colloca L, Amanzio M, Price DD, et al. 2014. The magnitude of nocebo effects in pain: A meta-analysis. Pain

Reicherts P, Gerdes AB, Pauli P, Wieser MJ. 2016. Psychological Placebo and Nocebo Effects rely on Expectation and Previous Experience. J Pain 17: 203-14

Schenk LA, Sprenger C, Geuter S, Buchel C. 2014. Expectation requires treatment to boost pain relief: an fMRI study. Pain 155: 150-7

Schmid J, Bingel U, Ritter C, Schedlowski M, Gramsch C, Forsting M, Elsenbruch S. 2015. Neural underpinnings of nocebo hyperalgesia in visceral pain: A fMRI study in healthy volunteers. Neuroimage 120: 114-22.

Smith PF. 2012. A note on the advantages of using linear mixed model analysis with maximal likelihood estimation over repeated measures ANOVAs in psychopharmacology: comment on Clark et al. (2012). J Psychopharmacol 26: 1605-7

Wager TD. 2005. The neural bases of placebo effects in pain. Curr Dir Psychol Sci 14: 175-79 
Figure captions:

Figure 1: Overview of the procedure. Green arrows: Subjective stress measure. Blue arrows: Startle measure; each startle test consisted of six startle trials. Dashed blue arrow: Startle habituation trials. $\mathrm{P}=$ Placebo. $\mathrm{NH}=$ Natural History. $\mathrm{NI}=$ Nocebo Information. NTMAN = Nocebo Temperature Manipulation. VAS = Visual Analog Scale. Pain level was measured during each pain stimulation.

Figure 2: Panel A: Startle amplitude shown as Z-scores. The error bars represent the standard error of the mean. Panel B: Stress reported on numerical rating scales (0-100). The error bars represent the standard error of the mean. Panel C: Pain intensity reported on $100 \mathrm{~mm}$ visual analog scale. The error bars represent the standard error of the mean. All panels: Tman = Temperature manipulation. Inf = Information. Dashed vertical lines indicate when the nocebo/placebo manipulation was performed.

Figure 3: Individual (ID 1-61) observed pain ratings in each group. Trials 1-2 = Pre-test. Trial $3=$ Temperature manipulation trial. Trial 4-5 = Post-tests.

Figure 4: Individual (ID 1-61) predicted pain ratings in each group based on the linear mixed model with Group, Trial, and Sex as factors.

The change in startle and stress (pre-test - drug information) were used as covariates. Trials 1-2 = Pre-test. Trial 3 = Temperature manipulation trial. Trial 4-5 = Post-tests. 


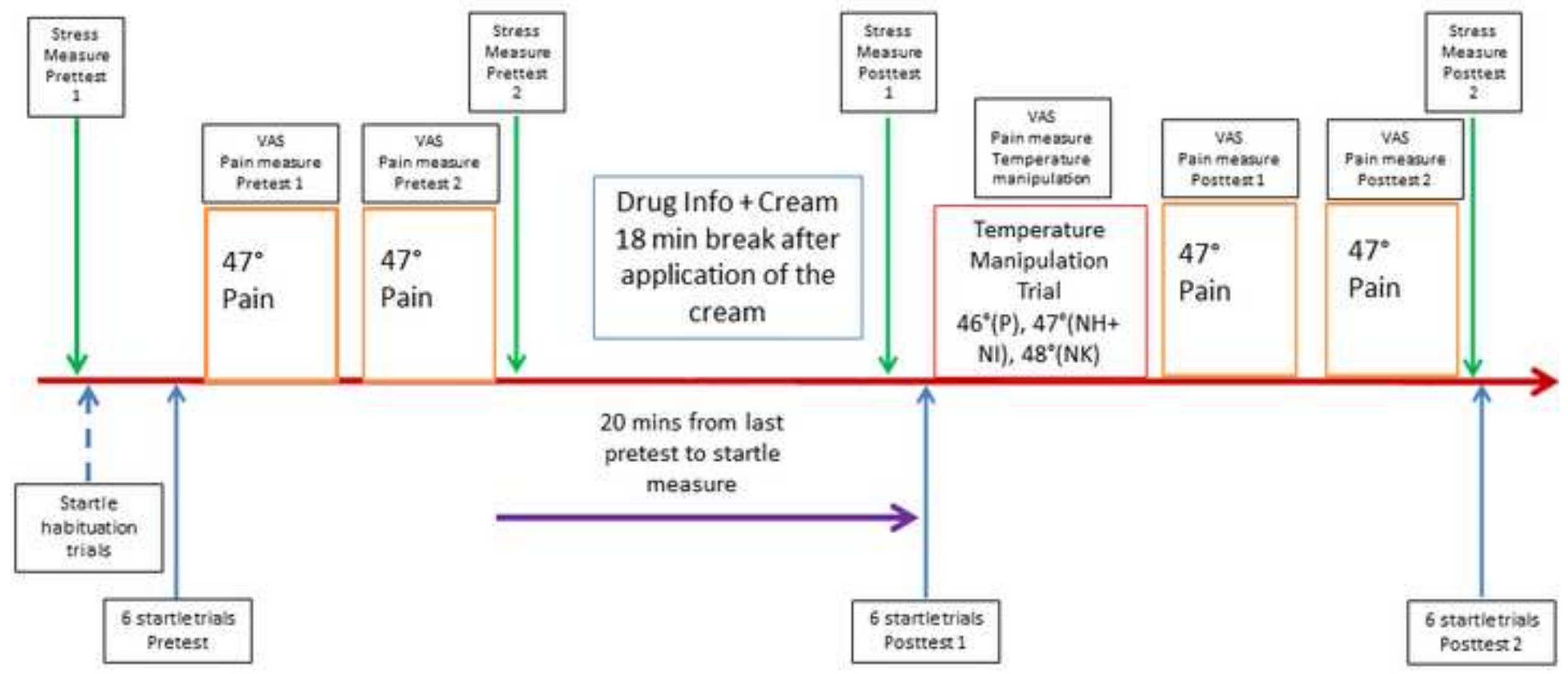


Figure 2

Click here to download high resolution image

A

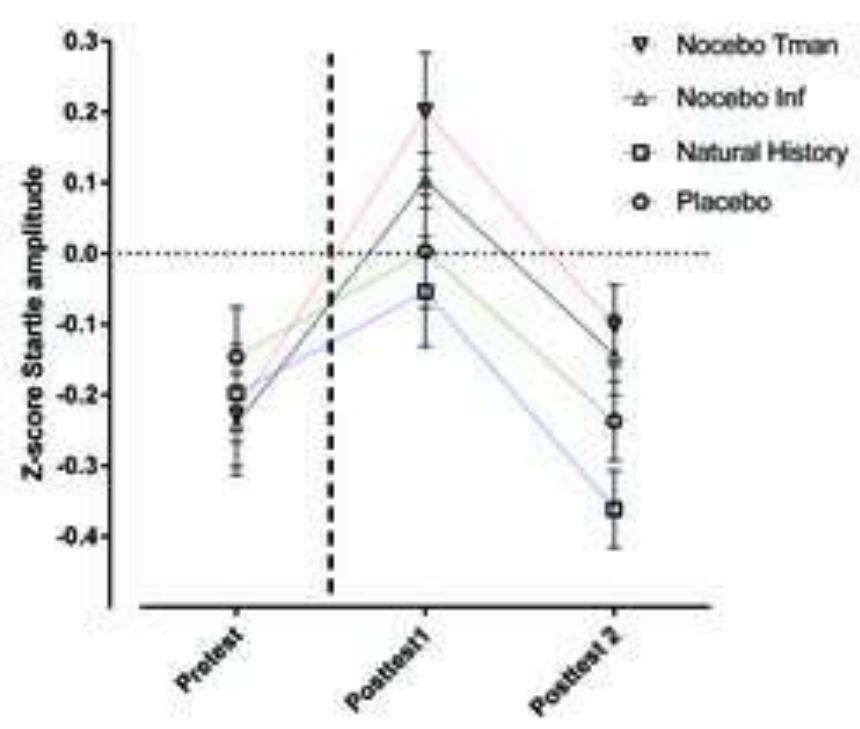

B

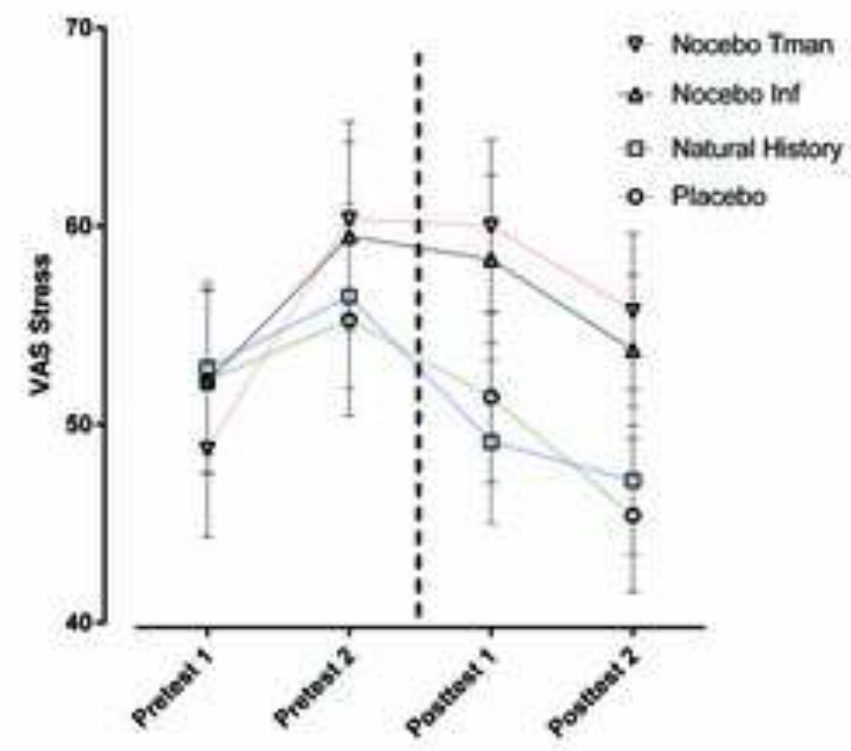

C

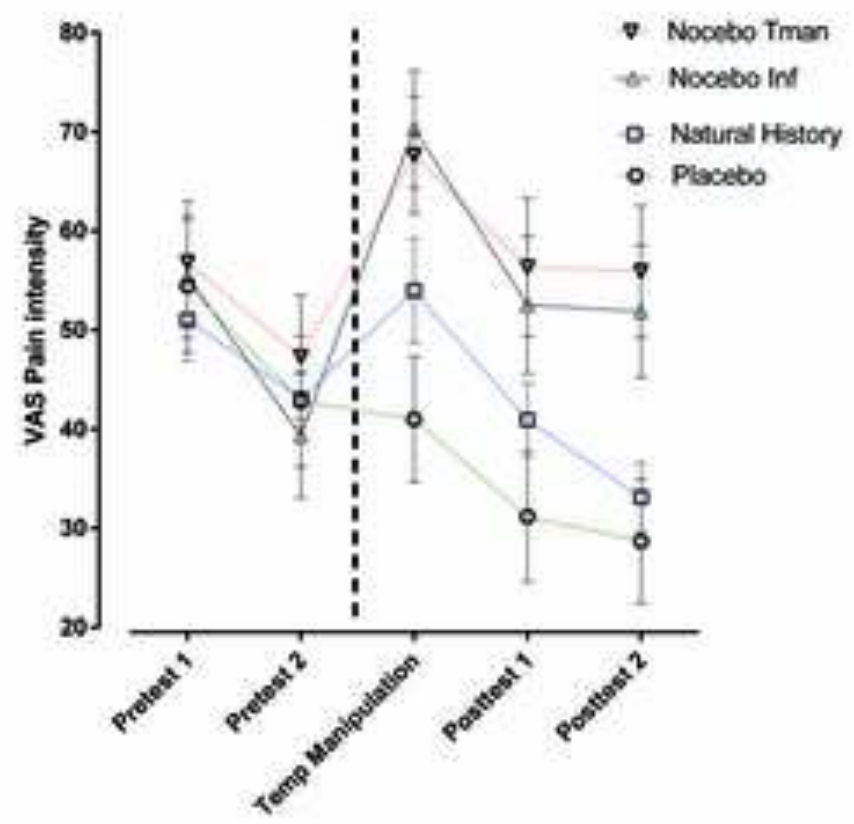




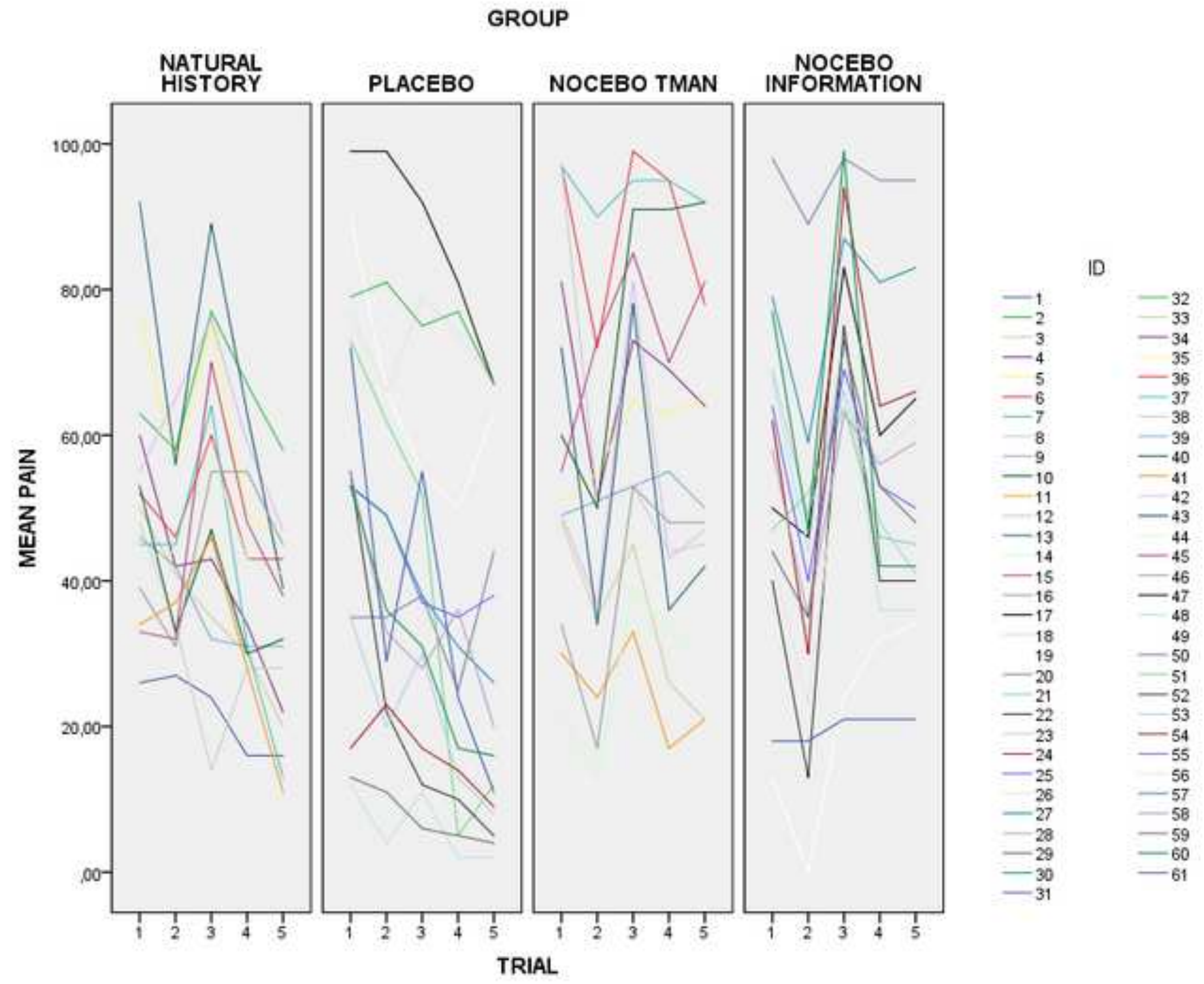




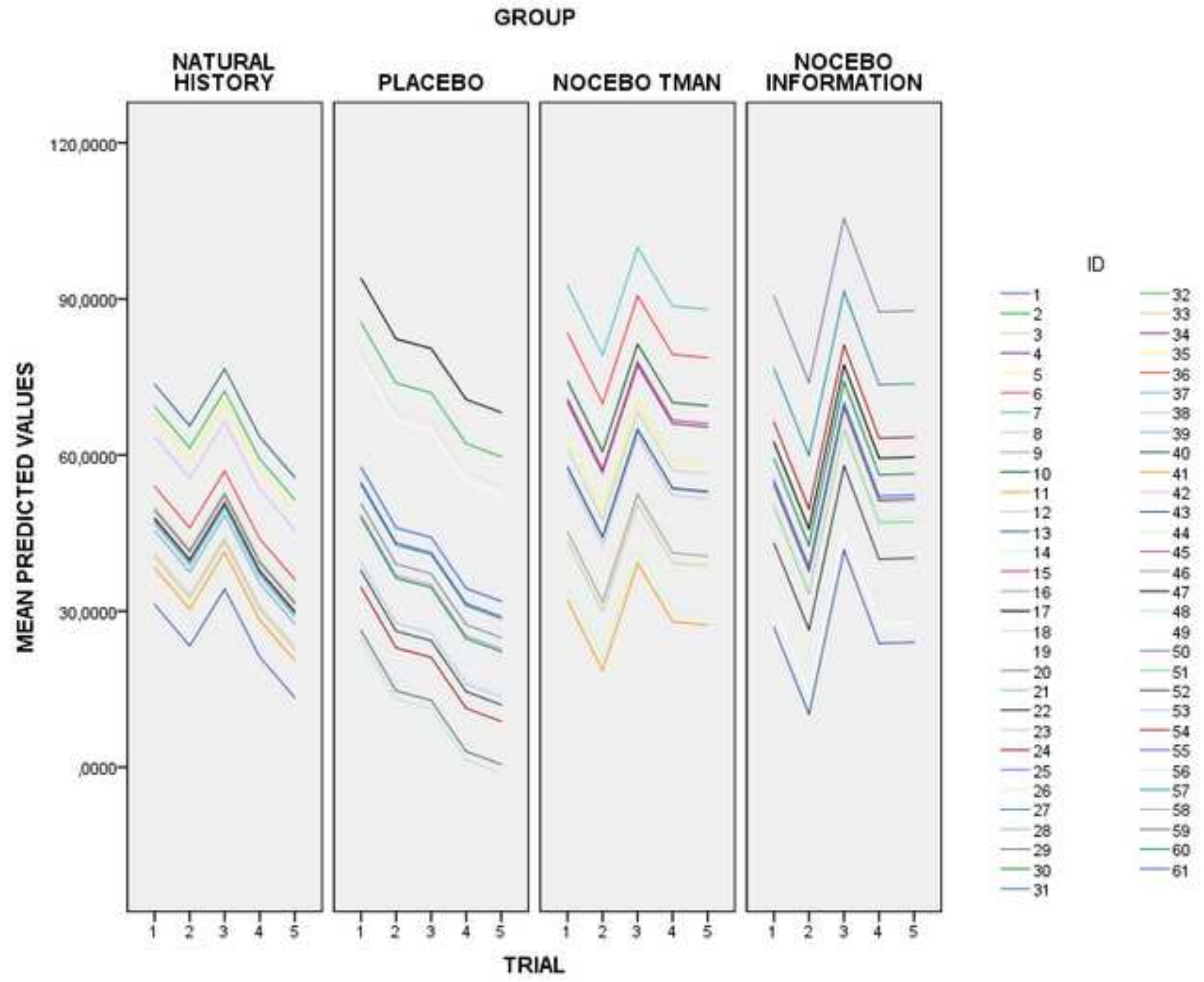

